\title{
Monitoring of Multilayered Bacterial Biofilm Morphology by Cryo-SEM for Raman Spectroscopy Measurements
}

\author{
K. Hrubanova ${ }^{1,2}$, S. Bernatova ${ }^{1}$, O. Samek ${ }^{1}$, M. Sery ${ }^{1}$, P. Zemanek ${ }^{1}$, J. Nebesarova ${ }^{3}$, F. Ruzicka ${ }^{4}$, \\ V. Krzyzanek ${ }^{1}$ \\ ${ }^{1 .}$ Institute of Scientific Instruments of the ASCR, v.v.i., Brno, Czech Republic. \\ 2. Brno University of Technology, Institute of Physical Engineering, Brno, Czech Republic. \\ 3. Biology center ASCR, Institute of Parasitology, Ceske Budejovice, Czech Republic. \\ 4. Department of Microbiology, Faculty of Medicine, Masaryk University and St. Anne's Faculty \\ Hospital, Brno, Czech Republic.
}

Staphylococcus epidermidis has been recently recognized as an important cause of serious biofilm infections associated with implanted medical devices. In presented work the multi-layered biofilms formed by these microorganisms were observed by scanning electron microscope (SEM), in particular with using freeze-fracturing technique. The freeze-fracture technique consists of physically breaking apart (fracturing) a rapidly frozen biological sample; structural detail exposed by the fracture plane is then visualized by metal deposition. An optional step, involving vacuum sublimation of ice, may be carried out after fracturing. Freeze fracture is unique among electron microscopic techniques in providing planar views of the internal organization of membranes or biofilms. Deep etching of ultrarapidly frozen samples permits visualization of the surface structure of cells and their components. Our sample was fractured after rapid-freezing into liquid nitrogen, the air-water was removed by short freeze-drying at Alto 2500 chamber (Gatan), sputtered by $5 \mathrm{~nm}$ Pt-Pd and finally imaged at low temperature in the field emission SEM JSM 7401F (JEOL).

When characterizing biofilms using spectroscopic techniques, and specifically Raman spectroscopy, a common approach is to analyze biofilm as a whole (cells embedded in extracellular matrix). Such spectra can be acquired point by-point at selected positions of individual colonies or using line-scan techniques such as e.g. Renishaw StreamLine. This approach collects data from many cells including the contribution from extracellular matrix. Therefore it is important to established contribution which translates to Raman spectra from extracellular matrix for reliable data presentation. This is crucial for understanding of the processes involved in cells embedded in the biofilm matrix. Such cells are well known to express phenotypes that differ from those of their planktonic counterparts. Moreover, they display specific properties including an increased resistance to chemical agent treatments. Raman spectroscopy employs a laser beam that is focused with a microscope objective in order to excite and collect Raman scattering from a small volume of the sample. A typical Raman spectrum from living microorganism contains a wealth of spectral peaks corresponding to unique interatomic vibrations in biomolecules e.g. nucleic acids, proteins, carbohydrates, and lipids [1].

In order to study how the extracellular matrix, that makes up the biofilm, translate into the Raman spectral "fingerprint" of the bacterial cells we have evaluated cryo-SEM image obtained from biofilmforming Staphylococcus epidermidis (Figure 1a). Using the detailed image (Figure 1b) we estimated that contribution of extracellular matrix is of about $40 \%$ of total volume. It means that when collecting Raman spectra we have not only contribution from bacterial cells of interest but also large contribution from the matrix. Thus, when analyzing cells embedded in extracellular matrix its contribution has to be taken into account to prevent misleading information about the cells. We show that combination of 
Raman spectroscopy with cryo-SEM can provide deeper inside into the chemistry and composition of biofilms. Such studies involving influence of variations in the amount of extracellular material - which depends on cultivations conditions and bacterial strain under investigation - are currently under way in our laboratories, exploiting combination of cryo-SEM and Raman spectroscopy techniques.

\section{References:}

[1] Bernatová, S. et al, Molecules 18 (2013), p. 13188.

[2] This work received support from the Ministry of Health, Ministry of Education, Youth and Sports of the Czech Republic (LO1212), together with the European Commission and the Czech Science Foundation (ALISI No. CZ.1.05/2.1.00/01.0017) and the Grant Agency of the Czech Republic (GA14-20012S).
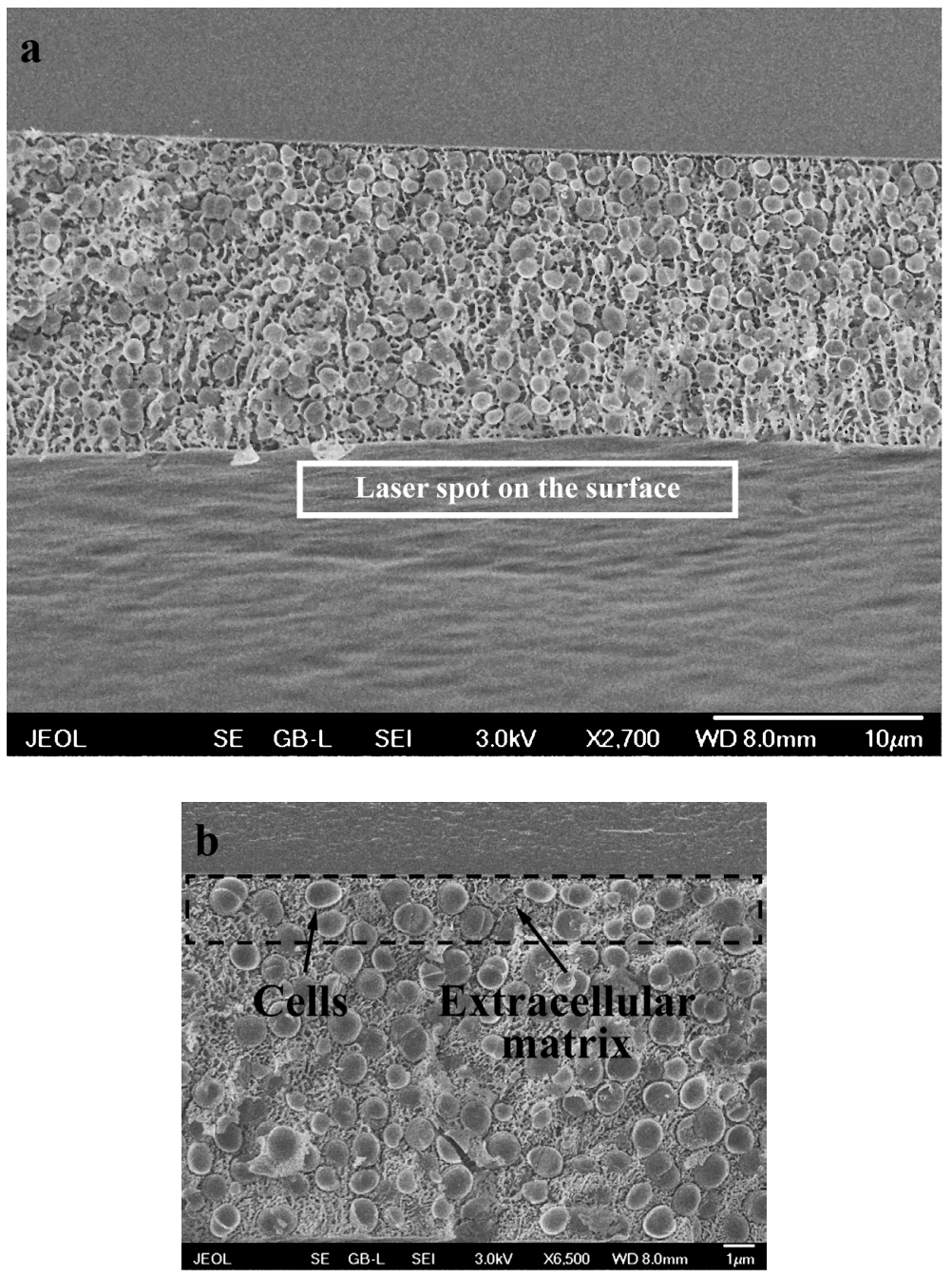

Figure 1. (a) cryo-SEM image of a biofilm-forming bacterial colony obtained by fracturing of biofilm on a glass coverslip. In order to demonstrate the influence of biofilm on Raman spectra the beam spot size employed for Raman spectroscopy analysis is shown (the beam spot size is of about $3 \times 20 \mu \mathrm{m}$ ). b) detail of the cells embedded in the extracellular matrix shows that the ratio of cells to extracellular matrix is about 60:40, respectively. 